\title{
RESEARCH
}

Open Access

\section{HBME1 and CK19 expression in non- invasive follicular thyroid neoplasm with papillary-like nuclear features (NIFTP) vs other follicular patterned thyroid lesions}

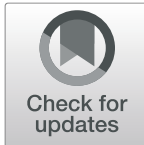

Qandeel Sadiq ${ }^{1}$, Radhika Sekhri ${ }^{2}$, Daniel T. Dibaba ${ }^{3,4}$, Qi Zhao $^{4}$ and Shweta Agarwal ${ }^{5^{*}}$ (1)

\begin{abstract}
Background: Thyroid neoplasms with follicular architecture can have overlapping morphologic features and pose diagnostic confusion among pathologists. Various immunohistochemical stains have been investigated as potential diagnostic markers for PTC, among which HBME1 and CK19 have gained popularity. Non-invasive follicular thyroid neoplasm with papillary-like nuclear features (NIFTP) poses similar diagnostic challenges with interobserver variability and is often misdiagnosed as adenomatoid nodule or follicular adenoma. This study aims to evaluate expression of HBME1 and CK19 in NIFTPs in comparison to other well-differentiated thyroid neoplasms and benign mimickers.

Method: Seventy-three thyroid cases diagnosed over a period of 3 years at Methodist University Hospital, Memphis, TN, USA, were included in this study: 9 NIFTP; 18 papillary thyroid carcinoma (PTC); 11 follicular variant of papillary thyroid carcinoma, invasive (I-FVPTC); 24 follicular adenomas (FA); and 11 multinodular goiters/adenomatoid nodules (MNG). A tissue microarray (TMA) was constructed and HBME1 and CK19 immunohistochemistry was performed.

Results: $77.8 \%$ of NIFTPs, $88.9 \%$ of PTCs, $81.8 \%$ of I-FVPTCS, $16.7 \%$ of FAs, and 18.2\% of MNGs showed HBME-1 expression. 66.7\% of NIFTPS, $83.3 \%$ of PTCs, $81.8 \%$ of I-FVPTCs, 33.3\% of FAs, and 45.4\% of MNGs expressed CK19. Difference in expression of HBME1 and CK19 was statistically significant for NIFTP vs FA (qualitative; $p<0.05$ ) and NIFTP vs MNG ( $p<$ 0.05). No statistically significant difference was found for HBME1 in NIFTP vs PTC (conventional and FVPTC), $p \geq 0.2$. Sensitivity of HBME1 and CK19 for NIFTP were 78\% and 67\%, 88\% each for PTC, and 89\% and 100\% for FVPTC, respectively, while specificity of HBME1 and CK19 for NIFTP were 53\% each, 62\% each for PTC, and $\sim 55 \%$ each for FVPTC. Conclusion: Our study indicated that HBME1 and CK19 are valuable markers in differentiating NIFTPs from morphologic mimics like follicular adenoma and adenomatoid nodules/multinodular goiter. While HBME1 and CK19 are both sensitive in diagnosing lesions with PTC-like nuclear features, CK19 stains a higher number of benign lesions in comparison to HBME1. No increase in sensitivity or specificity in diagnosis of NIFTP, PTC, or FVPTC was noted on combining the two antibodies.
\end{abstract}

Keywords: NIFTP, Papillary, Follicular, HBME1, CK19

* Correspondence: shagarwal@salud.unm.edu

${ }^{5}$ Department of Pathology, University of New Mexico School of Medicine, MSC08 4640, 1 University of New Mexico, Albuquerque, NM 87131, USA

Full list of author information is available at the end of the article

(c) The Author(s). 2021 Open Access This article is licensed under a Creative Commons Attribution 4.0 International License, which permits use, sharing, adaptation, distribution and reproduction in any medium or format, as long as you give appropriate credit to the original author(s) and the source, provide a link to the Creative Commons licence, and indicate if changes were made. The images or other third party material in this article are included in the article's Creative Commons licence, unless indicated otherwise in a credit line to the material. If material is not included in the article's Creative Commons licence and your intended use is not permitted by statutory regulation or exceeds the permitted use, you will need to obtain permission directly from the copyright holder. To view a copy of this licence, visit http://creativecommons.org/licenses/by/4.0/ The Creative Commons Public Domain Dedication waiver (http://creativecommons.org/publicdomain/zero/1.0/) applies to the data made available in this article, unless otherwise stated in a credit line to the data. 


\section{Introduction}

The rate of thyroid cancer in the USA is escalating rapidly with a reported increase in incidence of $3.6 \%$ per annum and the number of newly diagnosed cases going up to 56,430 yearly [1-3]. Papillary thyroid carcinoma (PTC) makes up most of the thyroid malignancies with follicular variant being the most common subtype [4]. The follicular variant of papillary thyroid carcinoma (FVPTC) has become the most common architectural pattern with the percentage rising exponentially from 18 to $57 \%$ in the last few decades [5]. FVPTC has two known subtypes - infiltrative and encapsulated, with the latter demonstrating an indolent behavior. The encapsulated form could be invasive or non-invasive [6-12]. Several studies reiterated that non-invasive form of encapsulated FVPTC (NI-EFVPTC) exhibited a behavior comparable to that of benign nodules and was being overtreated [13]. This led to the proposal of the new terminology "Noninvasive follicular thyroid neoplasm with papillary-like nuclear features (NIFTP)" by the Endocrine Pathology Society working group (ESPWG) for these tumors with indolent behavior [13, 14].

Follicular lesions of the thyroid often pose diagnostic dilemmas due to the morphologic resemblance and architectural similarities in benign and malignant lesions. There are studies citing the prevalent interobserver variability in the diagnosis of thyroid lesions. Saxen et al. reported a 58\% agreement among pathologists for thyroid tumors $[15,16]$. Similar findings have been noted in further studies, especially pertaining to the follicular lesions of thyroid [17-20]. With increasing diagnostic perplexity, the focus shifted to use of immunohistochemical markers to delineate benign from malignant lesions and distinguish the various follicular neoplasms [7, 21-23]. Various immunohistochemical (IHC) stains have been investigated as potential diagnostic markers for PTC, which include CK19, HBME1 (Hector Battifora Mesothelial-1), FN1 (fibronectin1), CITED1 (Cbp/p300-interacting transactivator with Glu/ Asp-rich carboxy-terminal domain, 1, also known as melanocyte-specific gene 1), and GAL3 (galectin3) [4, 17]. Among these, HBME1 and CK19 have gained popularity. HBME1 is a monoclonal antibody which is known to act against the microvillous surface of mesothelial cells and has shown to be expressed in thyroid malignancies, while negative in benign lesions [4, 22, 24-26]. CK19 has also proved useful in this regard, exhibiting strong and diffuse expression in thyroid malignancies and focal weak staining in benign nodules [22, 27, 28].

This study aims at investigating expression of these two biomarkers (HBME1 and CK19) in the commonly encountered benign and malignant thyroid nodules in a random and blinded manner. Furthermore, the purpose was to study effectiveness of these markers in differentiating challenging cases of NIFTP from benign entities like follicular adenoma (FA) and adenomatoid nodules. No molecular studies were performed as part of this study.

\section{Material and methods Case selection}

After obtaining approval of this retrospective study by International Review Board (IRB) of University of Tennessee Health Sciences Center (UTHSC), the Methodist University hospital database was queried for thyroid cases belonging to the following categories: multinodular goiter (MNG)/adenomatoid nodules, FA, papillary thyroid cancer (PTC), invasive form of follicular variant of papillary thyroid carcinoma (I-FVPTC), and non-invasive follicular thyroid neoplasm with papillarylike nuclear features (NIFTP).

Benign thyroid nodules with variably dilated follicles, absent thick capsule, and flattened to hyperplastic lining epithelium were included under the MNG/adenomatoid nodule category. Solitary encapsulated thyroid nodules, architecturally and cytologically different from surrounding gland and lacking nuclear features of PTC, were included as FAs in this study. Malignant thyroid cases with classical nuclear features of PTC (nuclear enlargement, elongation and overlapping, chromatin clearing, nuclear membrane irregularity, nuclear grooves, and nuclear pseudoinclusions) showing complex papillary architecture were categorized as PTC. Variants like hobnail, tall cell and diffuse sclerosing were also included in the PTC category.

Since this study aims to analyze the diagnostic performance of HBME-1 and CK19 in distinguishing follicular patterned lesions, we categorized FVPTC variant as a separate category. Cases of PTC with follicular growth pattern showing infiltration into the surrounding thyroid were included as FVPTC-I while NIFTP/encapsulated FVPTC cases were selected based on the revised, universally accepted, specific inclusion/exclusion criteria published in 2019 [6].

A total of 73 cases over a period of 3 years from 2016 to 2019 were identified. Clinical data including age, gender, and size of tumor was recorded for each case. Detailed review of H\&E slides was conducted by a Head and Neck Pathologist (SA) to characterize the tumors in each category. A change in the primary diagnosis in three cases was rendered as follows: Two cases of FVPT $C$ were reclassified on review: (1) as NIFTP, due to presence of a capsule and absence of invasion and (2) as PTC, due to presence of mixed papillary and follicular architecture, while one case previously diagnosed as NIFTP was altered to I-FVPTC (due to presence of infiltration), making the total number as follows: MNG ( $n=11$ ) including hyperplastic and adenomatoid nodules, FA $(n=24)$ [4 Hürthle cell/oncocytic type, 1 of clear cell 
and macro-follicular type each, and 18 microfollicular], PTC $(n=18)$ [usual $=13$, hobnail $=2$, focal tall cell features $=2$, diffuse sclerosing $=1]$, FVPTC $(n=11)$, and $\operatorname{NIFTP}(n=9)$.

\section{Tissue microarray (TMA)}

H\&E slides were reviewed by the pathologist to select the best possible area representative of the diagnosis for the TMA. Formalin-fixed paraffin-embedded tissue blocks were then utilized to construct 1-mm single cores $(n=73)$ using a semi-automated tissue microarrayer (Advanced Tissue Arrayer from Veridiam) to evaluate the immunohistochemical expression.

\section{Immunohistochemistry (IHC)}

IHC for HBME1 (Cell-Marque pre-dilute Clone HBME1) and CK-19 (Roche Pre-dilute Dispenser Clone $=\mathrm{A} 53-\mathrm{B} / \mathrm{A} 2.26)$ was performed on the TMA created from paraffin-embedded tissue blocks of 73 selected cases at PathGroup, TN. IHC staining of all the cores was then analyzed in a random and blinded fashion. HBME1 and CK19 were considered positive if more than $10 \%$ lesional cells showed membranous and membranous/cytoplasmic staining, respectively.

\section{Statistical analysis}

Positive/negative IHC results (HBME1/CK19) were taken as categorical variables and analyzed by Chi-square analysis; result was expressed as a percentage (qualitative). Categorical variables were summarized as count and percentage and compared between disease types using the $\chi^{2}$ test. The association between HBME1 and CK19 expression and disease as a binary outcome (NIFTP, PTC, FA, etc.) was conducted using the logistic regression analysis adjusting for demographic variables.

The following analyses were performed:

1. Expression of HBME1 and CK19 in NIFTP was compared to that observed in other welldifferentiated thyroid neoplasms and $p$ value was calculated.

2. HBME1 and CK19 were cross classified, and their expression (cumulative) was compared between two diagnoses (NIFTP vs PTC, NIFTP vs FA, NIFTP vs MNG, and NIFTP vs FVPTC) and $p$ value was calculated.
3. HBME1 and CK19 were cross classified, and their expression (cumulative) was compared between all diagnoses and $p$ value was calculated.

A $p$ value $\leq 0.05$ is interpreted as statistically significant. A $p$ value $>0.05$ is interpreted as not statistically significant.

In addition, sensitivity, specificity, and predictive values for HBME1 and CK19 in diagnosing NIFTP were calculated. The association between HBME1 and CK19 expression and disease types as a binary outcome (NIFTP, PTC, FA, etc.) was conducted using the logistic regression analysis adjusting for demographic variables. The receiver operating characteristics (ROC) curves were produced for the prediction of the outcomes by the HBME1 and CK19 adjusting for covariates. All the statistical analysis was conducted using $\mathrm{R}$ version 3.5.3 (11 March 2019).

\section{Results}

Demographic data and immunohistochemical expression Table 1 summarizes the demographic data along with tumor size for the cases included in this study $(n=73)$. Results for HBME1 and CK19 immunohistochemical expression (number and percentage of positive cases) in the different diagnostic categories are summarized in Table 2.

\section{Statistical analysis \\ Comparing NIFTP with each separate diagnoses}

1. FA. $16.7 \%$ expressed HBME1, while $33.3 \%$ showed positive expression for CK19 vs $77.8 \%$ and $66.7 \%$ for NIFTPs, respectively. The difference was found to be statistically significant for both antibodies ( $p=0.002$ for HBME1 and $p=0.02$ for CK19).

2. MNG. $18.2 \%$ expressed HBME1 while $45.4 \%$ showed positive staining for CK19. The difference was statistically significant in both percentage ( $p=0.008$, HBME1; $p=0.009$, CK19).

3. PTC. No statistically significant difference was found for either HBME1 or CK19 expression between PTC and NIFTP.

4. FVPTC. No statistically significant difference was observed.

Table 1 Demographic data and tumor size of thyroid neoplasms included in the study

\begin{tabular}{|c|c|c|c|c|c|}
\hline & NIFTP $(n=9)$ & PTC $(n=18)$ & FVPTC $(n=11)$ & FA $(n=24)$ & MNG $(n=11)$ \\
\hline F/M & $3.5: 1$ & $2: 1$ & $4.5: 1$ & $3: 1$ & $4.5: 1$ \\
\hline Age range (years) & $41-72$ & $23-80$ & $21-84$ & $33-72$ & $25-80$ \\
\hline Tumor size (range in cm) & $0.2-6.5$ & $0.1-7.0^{\mathrm{a}}$ & $0.3-4.5^{\mathrm{a}}$ & $0.6-6.2$ & $2.7-7.6^{\mathrm{a}}$ \\
\hline
\end{tabular}

atargest tumor nodule was considered in multifocal cases 
Table 2 Immunohistochemical expression (qualitative) of HBME1 and CK19 in various well-differentiated lesions

\begin{tabular}{llllll}
\hline Diagnostic category & Number of cases $(\boldsymbol{n})$ & HBME1+ $(\boldsymbol{X})$ & HBME1+ (\%) & CK19+ $(\boldsymbol{Y})$ & CK19+ \\
\hline NIFTP & 9 & 7 & 77.8 & 6 & 66.7 \\
PTC & 18 & 16 & 88.9 & 15 & 83.3 \\
FVPTC & 11 & 9 & 81.8 & 9 & 81.8 \\
FA & 24 & 4 & 16.7 & 8 & 33.3 \\
MNG & 11 & 2 & 18.2 & 5 & 45.4 \\
Total & 73 & 38 & N/A & 43 & N/A \\
\hline
\end{tabular}

$X$ number of cases showing HBME1 positivity, $Y$ number of cases showing CK-19 positivity

Figure 1 shows the histologic features and immunohistochemical expression of HBME-1 and CK19 in NIFTP vs FA and MNG. Figure 2 shows histologic features and the immunohistochemical expression of HBME-1 and CK19 in PTC and FVPTC.

\section{Cross classification of HBME1 and CK19 and two-way diagnosis}

This method of data analysis showed statistically significant results for NIFTP vs FA $(p=0.002)$ and NIFTP vs MNG $(p=0.005)$, while no significance was found for NIFTP vs PTC and/or NIFTP vs FVPTC.

\section{Cross classification of HBME1 and CK19 and all diagnosis}

This method showed a $p$ value of $<0.0001$, which indicated highly significant results. Figure 3 shows the distribution of all diagnoses with cross classification of HBME1 and CK19. It is quite evident from the bar diagram that benign diagnoses like FA and MNG are clustered on the left-hand side of the graph with most cases staining negative for both antibodies (HBME1-CK19: Neg-Neg) while PTC and NIFTP have a higher distribution along the right-hand side of the graph (HBME1-CK19: Pos-Pos).

\section{ROC curve analysis results}

Tables 3, 4, and 5 summarize the sensitivity, specificity, positive predictive value, and negative predictive value of HBME1 and CK19 (as calculated by the ROC curve analysis and DeLong's test) for NIFTP, PTC (classical), and I-FVPTC, respectively.

\section{Discussion}

Papillary thyroid carcinoma is usually a morphologic diagnosis with characteristic nuclear features such as large, overlapping, ground glass nuclei, nuclear grooves, and pseudo inclusions and rarely requires immunohistochemistry to confirm the diagnosis. Histologically, classic PTC and follicular variant are the two major low-risk subtypes of PTC with other high-risk variants like tall cell, diffuse sclerosing, and hobnail variants reported in literature $[29,30]$.

FVPTC encompasses a wide spectrum of morphology ranging from micro- to macro-follicular and diffuse growth pattern and could be encapsulated or infiltrative often creating diagnostic confusion with other follicular neoplasms. Tallini et al. [31] in 2017 published a detailed historical review of the emergence of the term "Follicular Variant of Papillary Thyroid Carcinoma". FVPTC was



Fig. 1 Representative histologic images of NIFTP, FA, and MNG $(\mathbf{a}, \mathbf{d}, \mathbf{g})$. $\mathbf{b}, \mathbf{e}, \mathbf{h}$ The differential expression of HBME1. $\mathbf{c}, \mathbf{f}, \mathbf{i}$ Expression of CK19 in NIFTP, FA, and MNG, respectively. Magnifications in $\mathbf{a}, \mathbf{d}, \mathbf{f}, \mathbf{g}$, and $\mathbf{i}$ are $\times 22$ and $\mathbf{c}$ and $\mathbf{e}$ are $\times 25$ while $\mathbf{b}$ and $\mathbf{h}$ are $\times 30$ 


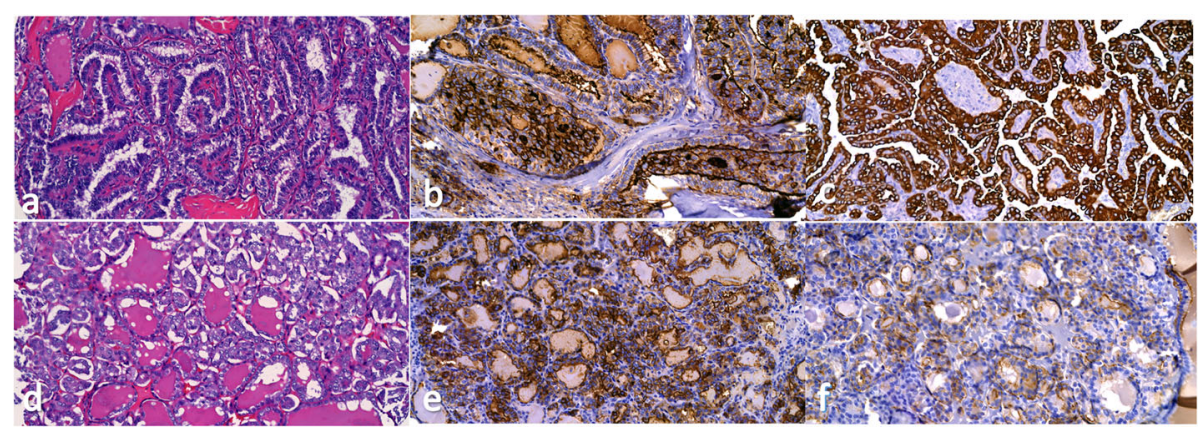

Fig. 2 Representative histologic images of PTC-c and FVPTC (a, d). b, e The differential expression of HBME1. $\mathbf{c}$, $\mathbf{f}$ Expression of CK19 in PTC and FVPTC, respectively. Magnifications in $\mathbf{a}$, c, and $\mathbf{d}$ are $\times 22$ and $\mathbf{f}$ is $\times 25$ while $\mathbf{b}$ and $\mathbf{e}$ are $\times 30$

first officially defined by Chen and Rosai [32] in 1977 after Lindsay found papillary carcinoma-like nuclear features in a subset of follicular carcinomas [29]. In 1980s, the encapsulated variant of FVPTC was recognized. This led to the classification of thyroid tumors showing predominant follicular growth pattern with nuclear characteristics of PTC into 3 main groups: (1) encapsulated FVPTC without invasion (EFVPTC), and (2) encapsulated FVPTC with capsular and/or vascular invasion and infiltrative FVPTC without a tumor capsule [31]. In 2016, non-invasive EFVPTC was re-categorized as non-invasive follicular thyroid neoplasm with papillarylike nuclear features (NIFTP) by Nikiforov et al. [13].

Since the new classification in 2016, several studies have evaluated biologic behavior of NIFTPs. Analysis of 94 NIFTP cases by Thompson et al. [33] and 129 cases by Rosario et al. [7] supported the low-risk behavior and conservative approach in treating the patients with NIFT P. Molecular studies on the encapsulated/well-circumscribed FVPTCs have found primarily RAS mutations and thereby suggested their close relationship with other follicular neoplasms of the thyroid such as follicular adenoma and follicular carcinoma [34-37].

Follicular patterned lesions of the thyroid have high level of interobserver as well as intraobserver disagreement [37, 38]. A considerable degree of discordance has been reported among pathologists in the diagnosis of FVPTC, the encapsulated type, in particular [20, 38]. The diagnostic criteria for NIFTP includes encapsulated/ well-demarcated tumor without any invasion, no papillary growth, no evidence of psammomatous calcifications or tumor necrosis, $<30 \%$ solid/trabecular or insular growth

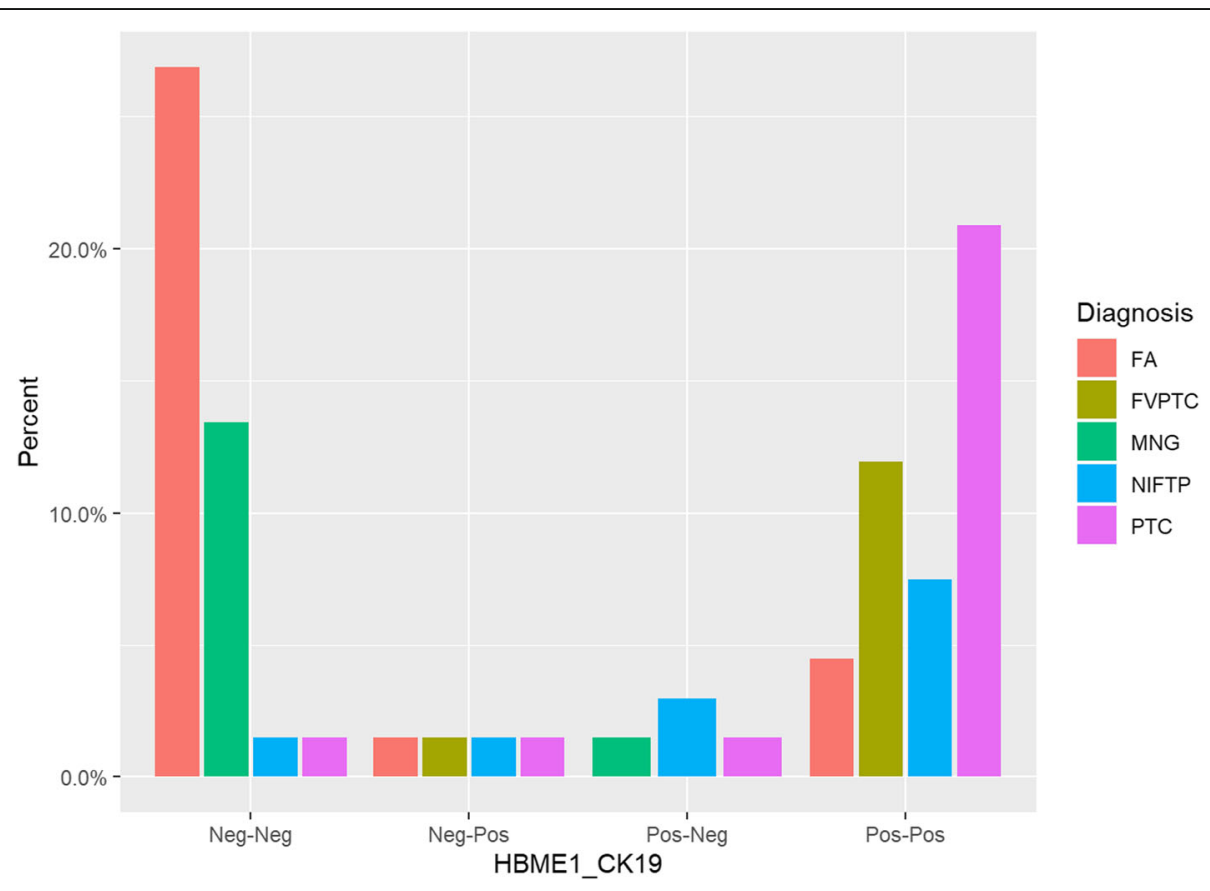

Fig. 3 Bar diagram showing all diagnosis and cross classification of HBME1 and CK19 
Table 3 Sensitivity, specificity, and predictive values of HBME1 and CK19 in diagnosis of NIFTP

\begin{tabular}{lllll}
\hline & Sensitivity & Specificity & Positive predictive value & Negative predictive value \\
\hline HBME1 & 0.78 & 0.53 & 0.19 & 0.94 \\
CK19 & 0.67 & 0.53 & 0.18 & 0.91 \\
HBME1 + CK19 & 0.56 & 0.57 & 0.17 & 0.89 \\
\hline
\end{tabular}

pattern, and nuclear features of PTC with nuclear score of 2-3 [13, 34].

A high degree of interobserver variability has been observed, even among expert pathologists as the nuclear features of PTC could be only focal/subtle [13, 38]. Unfortunately, there are no established criteria like required percentage of the follicular neoplasm showing nuclear features of PTC and/or the more reliable nuclear features (overlapping vs irregular nuclear outlines) that can help diagnose this entity as EFVPTC vs FA [38, 39]. NIFTP is still an evolving diagnosis and the struggle in diagnosing this entity is real.

Immunohistochemistry, although seldom required, can be helpful in differentiating FVPTC from other follicular lesions [26, 40, 41]. Various IHC markers have been explored to characterize the immunohistochemical profile of thyroid tumors especially the follicular patterned lesion which causes significant diagnostic confusion with high rate of interobserver disagreement. Among these, most notable are HBME1, Cytokeratin 19 (CK19), galectin-3 (GAL3), CITED1, and Thyroid peroxidase (TPO). HBME1 (Hector Battifora Mesothelial-1), a monoclonal antibody directed against microvilli and a marker of mesothelial and other epithelial cells, has shown significant expression in malignant thyroid with a sensitivity of $78.8 \%$ for thyroid malignancy, $87.3 \%$ for PTC, and $65.2 \%$ for follicular carcinomas and specificity of $82.1 \%$ [37, 42]. In our study, sensitivity and specificity of HBME-1 for PTC was found to be $89 \%$ and $62 \%$, respectively, and $89 \%$ and $55 \%$ for FVPTC, respectively.

CK19 is a low molecular weight cytokeratin which is demonstrated in both simple as well as complex epithelium and has been widely utilized in thyroid neoplasms $[17,21,43]$. Baloch et al. [44] employed a panel of cytokeratins including CK5/6 and CK 18, 10/13, 14, 17, 18, 19, and 20 in FVPTC. The authors found that CK19 was useful in diagnosis of PTC (showed diffuse staining pattern), only focally expressed in follicular tumors, but was expressed in normal thyroid tissue. It has also been proven a helpful marker in cytology specimens of unequivocal cases of PTC [28, 45]. Khurana et al. [45] reported a sensitivity and specificity of $93 \%$ and $100 \%$ which was comparable to that reported by Nasser et al. [28]. In our series, the sensitivity and specificity of CK19 in diagnosis of PTC were $88 \%$ and $63 \%$, respectively, while the values were $100 \%$ and $57 \%$ for diagnosis of FVPTC. Although the staining was weak to moderate in intensity, we did see about 33\% FAs and 45\% MNG nodules showing CK19 expression. Our findings agree with those of Baloch et al. and Haiyan Lu et al. [37, 40] who also found CK19 expression in normal thyroid parenchyma. We did not find any difference in antigen localization among positive malignant vs positive benign cases. Similar to our findings, Casey et al. [46] also reported weak to moderate positive expression of CK19 in $12 / 30$ benign thyroid cases with papillary hyperplasia with a sensitivity and specificity of $100 \%$ and $60 \%$ for PTCs.

In our study, we found a significant difference in the expression of CK19 and HBME1 in NIFTP cases in comparison to other benign follicular lesions $(p<0.02$ for both markers). HBME1 was expressed in $77.8 \%$ cases of NIFTP, while only $16.7 \%$ and $18.2 \%$ cases of FA and MNG showed positive staining, respectively. Frequent expression was also noted in CPTC (88.9\%) and FVPTC $(81.8 \%)$ cases which agree with the percentage reported in literature [37, 47]. Gucer et al. [48] reported an expression score of $77 \%$ for HBME1 in non-invasive RAS like PTCS/NIFTPs, corroborating with our findings (77.8\% for NIFTPs in our study). Further, the sensitivity of HBME1 and CK19 in our study was found to be $78 \%$ and $67 \%$, respectively, for diagnosis of NIFTP, while specificity was $53 \%$ for both biomarkers. To the best of our knowledge, this is the first study evaluating diagnostic value of HBME-1 and CK19 in NIFTP in comparison to other benign and malignant follicular patterned neoplasms of the thyroid gland.

Similarly, CK19 showed higher expression in NIFTP (66.7\%), cPTC (83.3\%), and FVPTC (81.8\%) in comparison to FA (33.3\%) and MNG (45.4\%). Similar findings

Table 4 Sensitivity, specificity, and predictive values of HBME1 and CK19 in diagnosis of PTC

\begin{tabular}{lllll}
\hline & Sensitivity & Specificity & Positive predictive value & Negative predictive value \\
\hline HBME1 & 0.89 & 0.62 & 0.44 & 0.94 \\
CK19 & 0.88 & 0.63 & 0.44 & 0.94 \\
HBME1 + CK19 & 0.82 & 0.68 & 0.47 & 0.92 \\
\hline
\end{tabular}


Table 5 Sensitivity, specificity, and predictive values of HBME1 and CK19 in diagnosis of FVPTC

\begin{tabular}{lllll}
\hline & Sensitivity & Specificity & Positive predictive value & Negative predictive value \\
\hline HBME1 & 0.89 & 0.55 & 0.24 & 0.97 \\
CK19 & 1.0 & 0.57 & 0.26 & 1.0 \\
HBME1 + CK19 & 0.89 & 0.62 & 0.27 & 0.97 \\
\hline
\end{tabular}

have been reported by Liu et al. [49] who reported statistically significant expression of CK19 and HBME1 in PTC vs benign thyroid lesions. Sensitivity of CK19 and HBME1 in diagnosis of PTC was reported to be $96.30 \%$ and $85.3 \%$, respectively, while the reported specificity was $40 \%$ and $62 \%$, respectively [49]. Our series reported a sensitivity and specificity of $\sim 89 \%$ and $\sim 63 \%$, respectively, for both antibodies in diagnosis of PTC (Table 4). For FVPTC, the sensitivity of HBME1 and CK19 was found to be $89 \%$ and $100 \%$, respectively, while specificity was $\sim 55 \%$ for both antibodies (Table 5 ).

HBME1 was found to be the most sensitive marker of thyroid malignancy by Palo et al. [50], followed by CK19, in differentiating FVPTC from FA and follicular carcinoma. Palo et al. reported an increase in sensitivity with combined use of HBME1 and CK19 in differentiating benign from malignant thyroid lesions [ $94 \%$ with combined use vs 86\% (HBME1) and 75\% (CK19)]. Saleh et al. [47] did not report increase in sensitivity or specificity with combined use of CK19 and HBME1 vs isolated use of either biomarkers. Findings in our series were concordant with Saleh et al.'s findings and showed no increase in the sensitivity when combining the two antibodies.

Liu et al. [37] published a review article in 2015 in which they analyzed various studies evaluating role of $\mathrm{IHC}$ in diagnosing thyroid lesions. The authors concluded that there is no single biomarker sufficient to differentiate between benign and malignant thyroid lesions. Their review found strong and diffuse HBME1 expression, while CK19 had low sensitivity as well as specificity for papillary thyroid carcinomas. The authors suggested including TROP2 (trophoblastic cell surface antigen 2) in the panel along with HBME1, CK19, and Galectin-3 as an aid in diagnosis of PTCs. Our study showed almost similar sensitivity and specificity for HBME-1 and CK19 in diagnosis of PTC and FVPTC as well as NIFTP cases with no added benefit of combining the two antibodies. However, in agreement with Liu et al.'s findings, we found HBME-1 to be a better marker of PTC/FVPTC/ NIFTP than CK19, due to the latter showing positive expression in a significant percentage of benign cases (33\% FAs and 45\% MNG/adenomatoid nodules in this study).

Our study has some limitations, and the findings need further validation. First, our sample size is small with limited number of NIFTP cases $(n=9)$. Second, we recognize that the study used TMA for IHC analysis, and the results might not be completely generalizable as some of these lesions can exhibit heterogeneity for antigen expression.

\section{Conclusion}

Thyroid lesions with follicular architecture have several overlapping histologic features with problems arising particularly in differentiating encapsulated FVPTC/ NIFTP from follicular adenomas or adenomatoid nodules in MNG. Our study revealed that HBME1 and CK19 are sensitive markers for diagnosis of NIFTPs, PTC, and FVPTC and can help in rendering the correct diagnosis in challenging cases of EFVPTC without invasion and/or NIFTP with focally developed PTC-like nuclear features. Further, our statistical analysis did not find added significance of combining these two markers in aiding the diagnosis of NIFTP/PTC or FVPTC. We acknowledge that the sample size of this study is small and further studies with larger number of cases (particularly NIFTP) are needed to further validate the findings. Nevertheless, the entire tumor capsule interface should be examined to rule out capsular and/or vascular invasion to avoid missing diagnosis of invasive carcinoma.

\section{Abbreviations \\ SA: Shweta Agarwal; QS: Qandeel Sadiq; RS: Radhika Sekhri; DTD: Daniel T Dibaba; QZ: Qi Zhao; NIFTP: Non-invasive follicular thyroid neoplasm with papillary-like nuclear features; TMA: Tissue microarray; MNG: Multinodular goiter; FA: Follicular adenoma; PTC: Papillary thyroid carcinoma; FVPT C: Follicular variant of papillary thyroid carcinoma; \\ IHC: Immunohistochemistry; Pos: Positive; Neg: Negative}

\section{Acknowledgements}

None

\section{Authors' contributions}

SA designed the study, selected the blocks for tissue microarray (TMA), read and interpreted the immunohistochemistry, supervised the work, and edited the final manuscript. QS helped collect the cases for study and helped in data collection and RS helped with TMA preparation. QS and RS contributed equally towards drafting the manuscript. DTD and QZ analyzed the raw data using statistical analysis. The authors read and approved the final manuscript.

\section{Funding}

The cost for immunohistochemistry (HBME1 and CK19) performed at PathGroup laboratories was paid with faculty development funds (SA).

\section{Availability of data and materials}

All data generated or analyzed during this study are included in this published article. 


\section{Declarations}

\section{Ethics approval and consent to participate}

The study was reviewed and approved by the International Review Board (IRB) at the University of Tennessee Health Sciences Center (UTHSC).

\section{Consent for publication}

Not applicable.

\section{Competing interests}

The authors declare that they have no competing interests.

\section{Author details}

'Department of Pathology, Methodist University Hospital, University of Tennessee Health Sciences Center, Memphis, TN, USA. ${ }^{2}$ Department of Pathology, Montefiore Medical Center/Albert Einstein College of Medicine, New York City, NY, USA. ${ }^{3}$ Tennessee Clinical and Translational Science Institute, University of Tennessee Health Science Center, Memphis, TN, USA ${ }^{4}$ Department of Preventive Medicine, University of Tennessee Health Science Center, Memphis, TN, USA. ${ }^{5}$ Department of Pathology, University of New Mexico School of Medicine, MSC08 4640, 1 University of New Mexico, Albuquerque, NM 87131, USA.

Received: 22 February 2021 Accepted: 30 April 2021

Published online: 08 May 2021

\section{References}

1. Xu B, Reznik E, Tuttle RM, Knauf J, Fagin JA, Katabi N, et al. Outcome and molecular characteristics of non-invasive encapsulated follicular variant of papillary thyroid carcinoma with oncocytic features. Endocrine. 2019 Apr; 64(1):97-108. https://doi.org/10.1007/s12020-019-01848-6.

2. Lim H, Devesa SS, Sosa JA, Check D, Kitahara CM. Trends in Thyroid Cancer Incidence and Mortality in the United States, 1974-2013. JAMA. 2017; 317(13):1338-48. https://doi.org/10.1001/jama.2017.2719.

3. Seigel RL, Miller KD, Jemal A. Cancer statistics, 2018. CA Cancer J Clin. 2018; 68(1):7-30.

4. Nasr MR, Mukhopadhyay S, Zhang S, Katzenstein AL. Immunohistochemical markers in diagnosis of papillary thyroid carcinoma: Utility of HBME1 combined with CK19 immunostaining. Mod Pathol. 2006;19(12):1631-7. https://doi.org/10.1038/modpathol.3800705.

5. Jung CK, Little MP, Lubin JH, Brenner AV, Wells SA, Sigurdson AJ, et al. The increase in thyroid cancer incidence during the last four decades is accompanied by a high frequency of BRAF mutations and a sharp increase in RAS mutations. J Clin Endocrinol Metab. 2014;99(2):E276-85. https://doi. org/10.1210/jc.2013-2503.

6. Rossi ED, Faquin WC, Baloch Z, Fadda G, Thompson L, Larocca LM, et al. Noninvasive Follicular Thyroid Neoplasm with Papillary-Like Nuclear Features (NIFTP): Update and Diagnostic Considerations-a Review. Endocr Pathol. 2019;30(2):155-62. https://doi.org/10.1007/s12022-019-9574-7.

7. Rosario PW, Mourao GF, Nunes MB, Nunes MS, Calsolari MR. Noninvasive follicular thyroid neoplasm with papillary like nuclear features. Endocr Relat Cancer. 2016;23(12):893-7. https://doi.org/10.1530/ERC-16-0379.

8. Xu B, Tallini G, Scognamiglio T, Roman BR, Tuttle RM, Ghossein RA. Outcome of large noninvasive follicular thyroid neoplasm with papillarylike nuclear features. Thyroid. 2017;27(4):512-7. https://doi.org/10.1089/ thy.2016.0649.

9. Parente DN, Kluijghout WP, Bongers PJ, Verzijl R, Devon KM, Rotstein LE, et al. Clinical safety of renaming encapsulated follicular variant of papillary thyroid carcinoma. Is NIFTP truly benign? World J Surg. 2018;42(2):321-6. https://doi.org/10.1007/s00268-017-4182-5.

10. Cho U, Mete O, Kim MH, Bae JS, Jung CK. Molecular correlates and rate of lymph node metastasis of non-invasive follicular thyroid neoplasm with papillary-like nuclear features and invasive follicular variant papillary thyroid carcinoma: The impact of rigid criteria to distinguish non-invasive follicular thyroid neoplasm with papillary-like nuclear features. Mod Pathol. 2017; 30(6):810-25. https://doi.org/10.1038/modpathol.2017.9

11. Alves VAF, Kakudo K, LiVolsi V, Lloyd RV, Nikiforov YE, Nose V, et al. Noninvasive follicular thyroid neoplasm with papillary-like nuclear features (NIFTP): achieving better agreement by refining diagnostic criteria. Clinics (Sao Paulo). 2018;73:e576.
12. Kakudo K, El-Naggar AK, Hodak SP, Khanafshar E, Nikiforov YE, Nose V, et al. Noninvasive follicular thyroid neoplasm with papillary-like nuclear features (NIFTP) in thyroid tumor classification. Pathol Int. 2018;68(6):327-33. https:// doi.org/10.1111/pin.12673.

13. Nikiforov YE, Seethala RR, Tallini G, Baloch ZW, Basolo F, LDR T, et al. Nomenclature revision for encapsulated follicular variant of papillary thyroid carcinoma: a paradigm shift to reduce overtreatment of indolent tumors. JAMA Oncol. 2016;2(8):1023-9.

14. O'Hare K, O'Regan E, Khattak A, Healy ML, Toner M. Reclassification as NIFTP: a retrospective review in a single institution with an emphasis on workload. Endocr Pathol. 2018;29(3):231-5. https://doi.org/10.1007/s12022-018-9538-3.

15. Lloyd RV, Asa SL, LiVolsi VA, Sadow PM, Tischler AS, Ghossein RA, et al. The evolving diagnosis of noninvasive follicular thyroid neoplasm with papillarylike nuclear features (NIFTP). Hum Pathol. 2018;74:1-4. https://doi.org/10.101 6/j.humpath.2017.12.027.

16. Saxén E, Franssila K, Bjarnason O, Normann T, Ringertz N. Observer variation in histologic classification of thyroid cancer. Acta Pathol Microbiol Scand Sect A. 1978;86A(6):483-6.

17. Scognamiglio T, Hyjek E, Kao J, Chen YT. Diagnostic usefulness of HBME1, galectin 3, CK19, and CITED1 and evaluation of their expression in encapsulated lesions with questionable features of papillary thyroid carcinoma. Am J Clin Pathol. 2006;126(5):700-8. https://doi.org/10.1309/044 V86JN2W3CN5YB.

18. Hirokawa M, Carney JA, Goellner JR, DeLellis RA, Heffess CS, Katoh R, et al. Observer variation of encapsulated follicular lesions of the thyroid gland. Am J Surg Pathol. 2002;26(11):1508-14. https://doi.org/10.1097/00000478-2 00211000-00014

19. Franc B. Observer variation of lesions of the thyroid. Am J Surg Pathol. 2003; 27(8):1177-9. https://doi.org/10.1097/00000478-200308000-00024

20. Lloyd RV, Erickson LA, Casey MB, Lam KY, Lohse CM, Asa SL, et al. Observer variation in the diagnosis of follicular variant of papillary thyroid carcinoma. Am J Surg Pathol. 2004;28(10):1336-40. https://doi.org/10.1097/01.pas. $0000135519.34847 . f 6$

21. Prasad ML, Pellegata NS, Huang Y, Nagaraja HN, Chapelle ADL, Kloos RT. Galectin-3, fibronectin-1, CITED-1, HBME1 and cytokeratin-19 immunohistochemistry is useful for the differential diagnosis of thyroid tumors. Mod Pathol. 2005;18(1):48-57. https://doi.org/10.1038/modpathol.3800235.

22. Saleh HA, Feng J, Tabassum F, Al-Zohaili O, Husain M, Giorgadze T. Differential expression of galectin-3, CK19, HBME1, and Ret oncoprotein in the diagnosis of thyroid neoplasms by fine needle aspiration biopsy. Cytojournal. 2009;6:18. https://doi.org/10.4103/1742-6413.55894.

23. Filie AC, Asa SL, Geisinger KR, Logani S, Merino M, Nikiforov YE, et al. Utilization of ancillary studies in thyroid fine needle aspirates: a synopsis of the National Cancer Institute Thyroid Fine Needle Aspiration State of the Science Conference. Diagn Cytopathol. 2008;36(6):438-41. https://doi.org/1 $0.1002 / d c .20831$

24. Sack MJ, Astengo-Osuna C, Lin BT, Battifora H, LiVolsi VA. HBME1 immunostaining in thyroid fine-needle aspirations: a useful marker in the diagnosis of carcinoma. Mod Pathol. 1997;10(7):668-74.

25. Saggiorato E, De Pompa R, Volante M, Cappia S, Arecco F, Tos APD, et al. Characterization of thyroid 'follicular neoplasms' in fine-needle aspiration cytological specimens using a panel of immunohistochemical markers: a proposal for clinical application. Endocr Relat Cancer. 2005;12(2):305-17. https://doi.org/10.1677/erc.1.00944

26. Asa SL. The role of immunohistochemical markers in the diagnosis of follicular-patterned lesions of the thyroid. Endocr Pathol. 2005;16(4):295-309. https://doi.org/10.1385/EP:16:4:295.

27. Erkiliç S, Koçer NE. The role of cytokeratin 19 in the differential diagnosis of true papillary carcinoma of thyroid and papillary carcinoma-like changes in Graves' disease. Endocr Pathol. 2005;16(1):63-6. https://doi.org/10.1385/EP:1 6:1:063.

28. Nasser SM, Pitman MB, Pilch BZ, Faquin WC. Fine-needle aspiration biopsy of papillary thyroid carcinoma: diagnostic utility of cytokeratin 19 immunostaining. Cancer. 2000;90(5):307-11. https://doi.org/10.1002/10970142(20001025)90:5<307::AID-CNCR7>3.0.CO;2-N.

29. Nath MC, Erickson LA. Aggressive variants of papillary thyroid carcinoma: hobnail, tall cell, columnar, and solid. Adv Anat Pathol. 2018;25(3):172-9. https://doi.org/10.1097/PAP.0000000000000184.

30. Agarwal S, Sadiq Q, Ortanca I. Hobnail cells in encapsulated papillary thyroid carcinoma: report of 2 cases with immunohistochemical and molecular 
findings and literature analysis. Pathol Res Pract. 2020;216(3):152678. https:// doi.org/10.1016/j.prp.2019.152678.

31. Tallini G, Tuttle RM, Ghossein RA. The history of the follicular variant of papillary thyroid carcinoma. J Clin Endocrinol Metab. 2017;102(1):15-22. https://doi.org/10.1210/jc.2016-2976.

32. Chem KT, Rosai J. Follicular variant of thyroid papillary carcinoma: a clinicopathologic study of six cases. Am J Surg Pathol. 1977;1(2):123-30. https://doi.org/10.1097/00000478-197706000-00003.

33. Thompson LD. Ninety-four cases of encapsulated follicular variant of papillary thyroid carcinoma: a name change to noninvasive follicular thyroid neoplasm with papillary-like nuclear features would help prevent overtreatment. Mod Pathol. 2016;29(7):698-707. https://doi.org/10.1038/modpathol.2016.65.

34. Seethala RR, Baloch ZW, Barletta JA, Khanafshar E. Non-invasive follicular thyroid neoplasm with papillary-like nuclear features: a review for pathologists. Modern Pathol. 2018:31(1):39-55. https://doi.org/10.1038/modpathol.2017.130.

35. Cancer Genome Atlas Research Network. Integrated genomic characterization of papillary thyroid carcinoma. Cell. 2014;159:676-90.

36. Howitt BE, Jia Y, Sholl LM, Barletta JA. Molecular alterations in partiallyencapsulated or well-circumscribed follicular variant of papillary thyroid carcinoma. Thyroid. 2013;23(10):1256-62. https://doi.org/10.1089/thy.2013.0018.

37. Liu H, Lin F. Application of immunohistochemistry in thyroid pathology. Arch Pathol Lab Med. 2015;139(1):67-82.

38. Elsheikh TM, Asa SL, Chan JKC, DeLellis RA, Heffess CS, LiVolsi VA, et al. Interobserver and intraobserver variation among experts in the diagnosis of thyroid follicular lesions with borderline nuclear features of papillary carcinoma. Am J Clin Pathol. 2008;130(5):736-44. https://doi.org/10.1309/A JCPKPZQUVNARCCP.

39. Thompson LDR, Poller DN, Kakudo K, Gooding WE, Burchette R, Nikiforova Y, and Seethala RR. An international interobserver variability reporting of the nuclear scoring criteria to diagnose noninvasive follicular thyroid neoplasm with papillary-like nuclear features: a validation study. Endocr Pathol 2018; 29(3):242-249, DOI: 10.1007/s12022-018-9520-0.

40. Lee SE, Hang TS, Choi YL. Molecular profiling of papillary thyroid carcinoma in Korea with a high prevalence of BAFV600E mutation. Thyroid. 2017;27(6): 802-10. https://doi.org/10.1089/thy.2016.0547.

41. Nakamura N, Erickson LA, Jin L, Kajita S, Zhang H, Qian X, et al. Immunohistochemical separation of follicular variant of papillary thyroid carcinoma from follicular adenoma. Endocr Pathol. 2006;17(3):213-23. https://doi.org/10.1385/EP:17:3:213.

42. Baloch Z, Mete O, Asa SL. Immunohistochemical biomarkers in thyroid pathology. Endocr Pathol. 2018;29(2):91-112. https://doi.org/10.1007/s12022-018-9532-9.

43. Sahoo S, Hoda SA, Rosai J, DeLellis RA. Cytokeratin 19 immunoreactivity in the diagnosis of papillary thyroid carcinoma: a note of caution. Am J Clin Pathol. 2001;116(5):696-702. https://doi.org/10.1309/6D9D-7JCM-X4T5-NNJY.

44. Baloch ZW, Abraham S, Roberts S, LiVolsi VA. Differential expression of cytokeratins in follicular variant of papillary carcinoma: an immunohistochemical study and its diagnostic utility. Hum Pathol. 1999; 30(10):1166-71. https://doi.org/10.1016/50046-8177(99)90033-3.

45. Khurana KK, Truong LD, LiVolsi VA, Baloch ZW. Cytokeratin 19 immunolocalization in cell block preparation of thyroid aspirates. An adjunct to fine-needle aspiration diagnosis of papillary thyroid carcinoma. Arch Pathol Lab Med. 2003;127(5):579-83. https://doi.org/10.5858/2003-127-0579-CIICBP.

46. Casey MB, Lohse CM, Lloyd RV. Distinction between papillary thyroid hyperplasia and papillary thyroid carcinoma by immunohistochemical staining for cytokeratin 19, galectin-3, and HBME1. Endocr Pathol. 2003; 14(1):55-60. https://doi.org/10.1385/EP:14:1:55.

47. Saleh HA, Jin B, Barnwell J, Alzohaili O. Utility of immunohistochemical markers in differentiating benign from malignant follicular-derived thyroid nodules. Diagn Pathol. 2010;5(1):9. https://doi.org/10.1186/1746-1596-5-9.

48. Gucer H, Bagci P, Bedir R, Sehitoglu I, Mete O. The value of HBME1 and claudin-1 expression profile in the distinction of BRAF-like and RAS-like phenotypes in papillary thyroid carcinoma. Endocr Pathol. 2016;27(3):22432. https://doi.org/10.1007/s12022-016-9433-8.

49. Liu Z, Yu P, Xiong Y, Zeng W, Li X, Maiaiti Y, et al. Significance of CK19, TPO, and HBME1 expression for diagnosis of papillary thyroid carcinoma. Int J Clin Exp Med. 2015;8(3):4369-74 eCollection 2015.

50. Palo S, Biligi DS. Differential diagnostic significance of HBME1, CK19 and S100 in various thyroid lesions. Malaysian J Pathol. 2017:39(1):55-67.

\section{Publisher's Note}

Springer Nature remains neutral with regard to jurisdictional claims in published maps and institutional affiliations.
Ready to submit your research? Choose BMC and benefit from:

- fast, convenient online submission

- thorough peer review by experienced researchers in your field

- rapid publication on acceptance

- support for research data, including large and complex data types

- gold Open Access which fosters wider collaboration and increased citations

- maximum visibility for your research: over $100 \mathrm{M}$ website views per year

At BMC, research is always in progress.

Learn more biomedcentral.com/submissions 